\title{
uvby FCAPT photometry of the mCP stars HR 2258, MW Vul, and HR 9017 and the HgMn star $46 \rho$ Aqr $^{\star}$
}

\author{
S. J. Adelman and K. J. Young \\ Department of Physics, The Citadel, 171 Moultrie Street, Charleston, SC 29409, USA \\ e-mail: adelmans@citadel.edu \\ Received 19 April 2004 / Accepted 28 August 2004

\begin{abstract}
Differential Strömgren uvby observations from the Four College Automated Photoelectric Telescope are presented for the magnetic CP (mCP) stars HR 2258, MW Vul, and HR 9017 and the HgMn star $46 \rho$ Aqr. A period for HR 2258 of 15.0313 days satisfactorily phases photometry over a 30 year time span. Recent observations of MW Vul agree in phasing with those taken 13 years ago, but the amplitudes in $u, v, b$, and $y$ are greater and the light curves have changed shape especially in the falling branch. This type of behavior has not been seen before in any slowly rotating $\mathrm{mCP}$ star. It is a signature of the precession of the rotational axis which brings different parts of the surface into view. A period of 0.92738 days is found for HR 9017 which is similar to that derived by Winzer. Changes in the v-c values between observations taken in the Spring and Fall of 2003 for $46 \rho$ Aqr may be due to its companion.
\end{abstract}

Key words. stars: chemically peculiar - stars: individual: HR 2258 - stars: individual: MW Vul stars: individual: $46 \rho$ Aqr - stars: individual: HR 9017

\section{Introduction}

Single-channel differential Strömgren $u v b y$ photometry from the Four College Automated Photoelectric Telescope (FCAPT) at Washington Camp, AZ, was obtained for the magnetic Chemically Peculiar (mCP) stars HR 2258, MW Vul, and HR 9017 and the Mercury-Manganese (HgMn) star $46 \rho$ Aqr. These investigations continue the studies of the stability of non-magnetic $\mathrm{CP}$ stars and of the photometric variability of $\mathrm{mCP}$ stars to determine both their rotational periods and the shapes of their light curves. Information on this paper's variables is summarized in Table 1 (Hoffleit 1982; Hoffleit et al. 1993). The automated telescope measures the dark count and then in each filter sky-ch-c-v-c-v-c-v-c-ch-sky for each group of variable (v), check (ch), and comparison (c) stars where sky is a reading of the sky. No corrections were made for any neutral density filter differences among the stars of each group. The comparison and check stars were chosen from presumably nonvariable stars using Hipparcos photometry (ESA 1997) that are located near the variables on the sky that had similar $V$ magnitudes and $B-V$ colors. The periods of the $\mathrm{mCP}$ stars were found with the Scargle periodogram (Scargle 1982; Horne \& Baliunas 1986).

* Tables 2-4 and 6 are only available in electronic form at the CDS via anonymous ftp to cdsarc.u-strasbg.fr (130.79.128.5) or via

http://cdsweb.u-strasbg.fr/cgi-bin/qcat?J/A+A/429/317
Table 1. Photometric groups.

\begin{tabular}{rllll}
\hline \hline HD & Star name & Type & $V$ & Spectral type \\
\hline 43819 & HR 2258 & v & 6.32 & B8IIIpSiCr \\
43153 & 72 Ori & c & 5.30 & B7V \\
41040 & 64 Ori & ch & 5.14 & B8III \\
& & & & \\
192913 & MW Vul & v & 6.68 & A0pSi \\
191747 & 18 Vul & c & 5.52 & A3III \\
192518 & 21 Vul & ch & 5.18 & A7IVa \\
& & & & \\
211838 & $46 \rho$ Aqr & v & 5.37 & B8IIIpMn:Hg \\
213320 & $\sigma$ Aqr & c & 4.82 & A0IVs \\
212404 & 51 Aqr & ch & 5.78 & A0V \\
& & & & \\
223358 & HR 9017 & v & 6.41 & A0pSrSi:Cr: \\
223624 & BD $+63^{\circ} 2064$ & c & 6.82 & A1V \\
225289 & HR 9110 & ch & 5.80 & B8pHgMn \\
\hline
\end{tabular}

Note on type of star: $\mathrm{v}=$ variable, $\mathrm{c}=$ comparison star, and $\mathrm{ch}=$ check star.

\section{Three magnetic CP stars}

The mCP stars are photometric, spectrum, and magnetic variables with emergent energy distributions, photospheric abundances, and magnetic field strengths changing with 


\section{HR 2258}
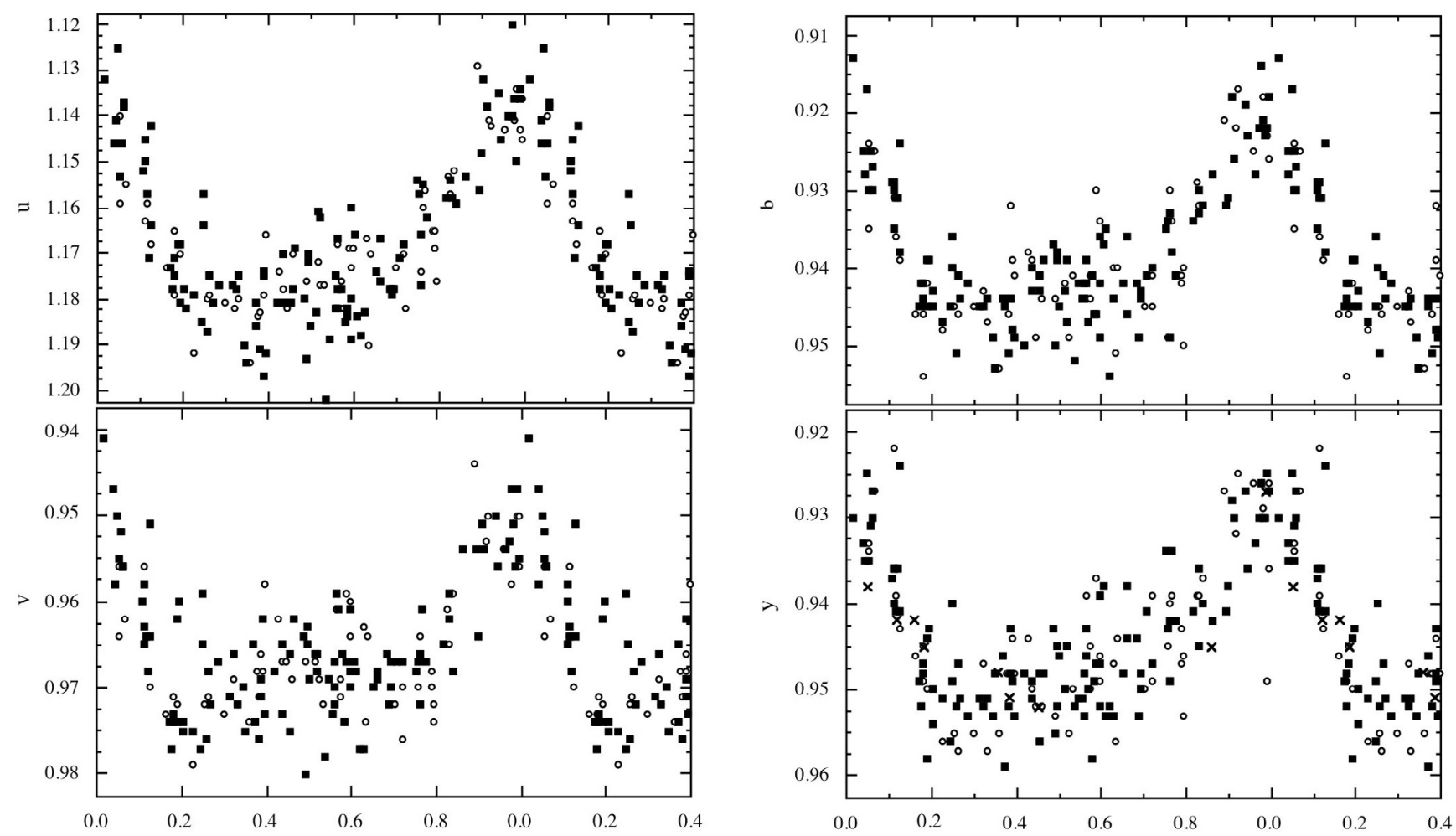

phase

Fig. 1. uvby photometry of the mCP star HR 2258 as a function of the ephemeris HJD(maximum light) $=2441253.709+15.0313 E$. The solid squares and the open circles in each panel are respectively values from Adelman (1997) and this paper. The $V$ values of Winzer (1974) transformed to $y$ are plotted as $x$ 's.

photospheric location. Since their magnetic and rotational axes are usually not aligned, a distant observer sees a variety of variable behavior as they rotate. Their anomalous photospheric abundances, currently thought to be produced by hydrodynamical processes in particular radiative diffusion and gravitational settling in radiative envelopes containing strong magnetic fields, are functions of the local magnetic field strength and the time since the star was on the Zero Age Main Sequence (Michaud \& Proffitt 1993, and references therein). Differential photometric studies with the FCAPT by the senior author and his students (see, e.g., Adelman 2002) have improved their periods and light curves so that they can be used to better relate observations taken at different times, detect variable light curves, and study the period distribution of the mCP stars. Surface maps of abundances for spectrum variables exhibiting moderate rotation can be derived to serve as tests of mCP star theories.

\section{1. $H R 2258$}

Winzer (1974) discovered the photometric variability of the sharp-lined $\left(v \sin i=14 \mathrm{~km} \mathrm{~s}^{-1}\right)$ star (Wolff \& Preston 1978) HR 2258 (HD 43819, V1155 Ori, HIP 33019). Adelman $(1975,1982)$ found it had a definite $\lambda 5200$ broad, continuum feature. Lopez-Garcia \& Adelman (1994) determined that most of its iron peak elemental abundances are 10 times solar while its rare earths are usually 1000 times solar. Adelman (1997) obtained 120 sets of Strömgren $u v b y$ measures with the FCAPT between years $2-6$ of its operation. With them a period of 15.0305 days was found. The asymmetric light curves with the fall from maximum being faster than the rise from minimum can be described as roughly in phase with amplitudes of $0.06 \mathrm{mag}$ in $u, 0.035 \mathrm{mag}$ in $b$, and $0.03 \mathrm{mag}$ in $v$ and $y$.

In the 2003-04 observing season of the FCAPT (year 14) 61 sets of Strömgren $u v b y$ values were obtained with the same comparison and check stars as used by Adelman (1997) (see Table 2). Between the two sets of data, the photometer was changed as were the neutral density filters. We can thus compare the two sets of data, but have to adjust the zero points to get the best overlap. In doing so, we made the average values agree.

When a periodogram was run on the entire data set, a slight adjustment in the period was found to be necessary. This also made a minor difference in the value for the initial JD. The new ephemeris is

$\mathrm{HJD}($ maximum light $)=2441253.709( \pm 0.025)+15.0313$ $( \pm 0.0002) E$.

Figure 1 shows the Adelman (1997) values as solid squares and the values from this paper as open circles plotted according to this ephemeris. The Strömgren data sets coincide reasonably well. Also shown in the $y$ panel are the $V$ values of Winzer (1974) transformed to $y$. They agree with the other data. 
MW Vul
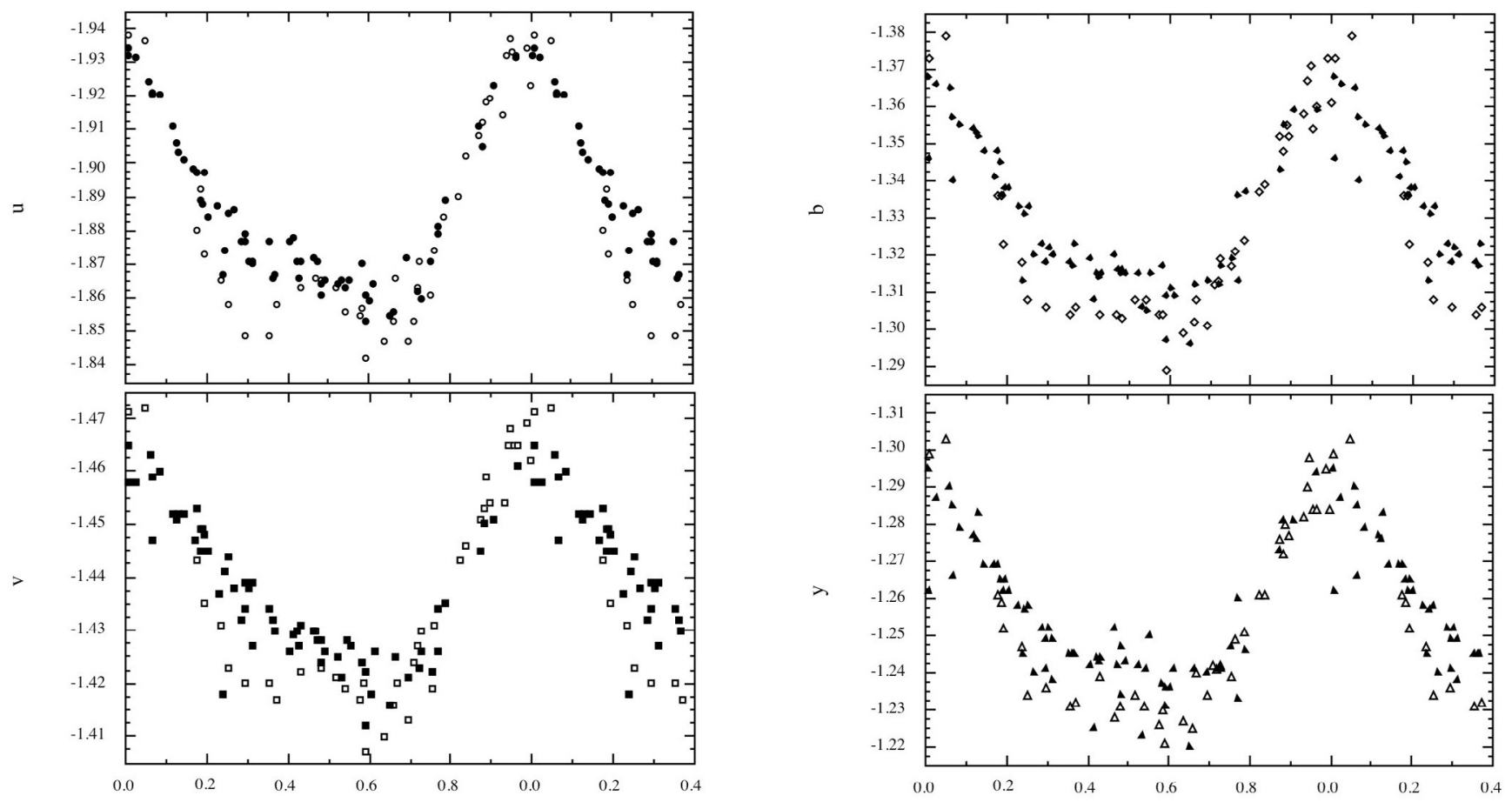

phase

Fig. 2. uvby photometry of the $\mathrm{mCP}$ star MW Vul as a function of the ephemeris HJD (maximum light) $=2441617.165+16.840 E$. The solid and open symbols in each panel are respectively values from year 1 (1990-91) and year 14 (2003-04) of the FCAPT. Differences in all light curves are seen near phase 0.3 and in the amplitude.

Thus for a time span of over 30 years HR 2258 has had a constant period.

\section{2. $M W$ Vul}

Preston (1971) found that MW Vul (HD 192913, HIP 99927 , $\left.\mathrm{BD}+27^{\circ} 3668\right)$ was one of the sharpest-lined Si stars $(v \sin i=$ $14 \mathrm{~km} \mathrm{~s}^{-1}$ ). It has a definite $\lambda 5200$ broad, continuum feature (Adelman 1982; Adelman \& Pyper 1993). Ryabchikova et al. (1990) studied its spectrum variability. Babcock (1958) reported a magnetic field of $483 \pm 221 \mathrm{G}$.

Adelman \& Knox (1994) determined a period of 16.840 days from their set of 87 FCAPT Strömgren photometry combined with the $V$ values of Winzer. This result is close to those of Bartolini et al. (1974) and Musielok et al. (1980).

In the 2002-2003 and the 2003-2004 observing seasons, we obtained respectively 13 and 41 new sets of Strömgren uvby observations (see Table 3). There are offsets in the numerical values as for HR 2258 (see above) between the two groups of observations. When the new values were plotted using the ephemeris of Adelman \& Knox (1994)

$\operatorname{HJD}($ maximum light $)=2441617.165+16.840 E$,

the light curves phase well with the previous $u v b y$ values indicating that the period was well determined. However the amplitudes of the light curves are somewhat different. Celestia 2000 (ESA 1998) gives a period of 16.829 days, but the baseline it uses was shorter. Adelman \& Knox (1994) connected their observations with those of Winzer (1974). There is a companion $0.210^{\prime \prime}$ away which is fainter by $2.5 \mathrm{mag}$.

Due to the variability in the amplitudes of the light curves, we decided to examine those with the most values separately. These are year 1 (1990-1991), year 2 (1991-1992), and year 14 (2003-2004) with 63, 27, and 41 sets of values, respectively. The shapes of the light curves from years 1 and 2 are similar with offsets of order $0.02 \mathrm{mag}$. To compare values from years 1 and 14 we added constants to the year 14 values so their means agreed with the year 1 means (see Fig. 2). In year 1 the amplitudes for $u, v, b$, and $y$ were $0.07,0.045$, 0.06 , and $0.06 \mathrm{mag}$, respectively, while in year 14 they have become $0.085,0.055,0.07,0.075 \mathrm{mag}$, respectively. In the falling branch, a secondary minimium near phase 0.3 has become more evident.

For several $\mathrm{mCP}$ stars similar observations have been explained as the result of the precession of the rotational axis about the magnetic axis. Several possible observational consequences follow: 1) if the star is rapidly rotating, it will be a flattened ellipsoid of rotation with its polar diameter smaller than its equatorial diameter. It will appear brighter when its polar axis more nearly points towards us; 2 ) the timings of the maximum, the minimum, and of any identifiable portion of their light curves will experience periodic changes during the precessional cycle as different parts of the inhomogenous photosphere come into and out of view; and 3) the observed value of $v \sin i$ will change, being smallest when the rotational pole is most nearly towards us. For the best documented case 56 Ari, 
Table 5. $\rho 46$ Aqr mean values.

\begin{tabular}{rcccccccc}
\hline \hline Season & $u(\mathrm{v}-\mathrm{c})$ & $u(\mathrm{ch}-\mathrm{c})$ & $v(\mathrm{v}-\mathrm{c})$ & $v(\mathrm{ch}-\mathrm{c})$ & $b(\mathrm{v}-\mathrm{c})$ & $b(\mathrm{ch}-\mathrm{c})$ & $y(\mathrm{v}-\mathrm{c})$ & $y(\mathrm{ch}-\mathrm{c})$ \\
\hline Spring 2003 & & & & & & & & \\
Average & 0.165 & -0.886 & 0.537 & -0.982 & 0.560 & -0.985 & 0.544 & -0.973 \\
$\sigma$ & 0.008 & 0.009 & 0.010 & 0.007 & 0.009 & 0.008 & 0.009 & 0.009 \\
& & & & & & & & \\
Average & 0.162 & -0.889 & 0.523 & -0.978 & 0.536 & -0.975 & 0.518 & -0.963 \\
$\sigma$ & 0.008 & 0.009 & 0.008 & 0.007 & 0.009 & 0.008 & 0.010 & 0.007 \\
\hline
\end{tabular}

Note on type: $\mathrm{v}=$ variable, $\mathrm{c}=$ comparison star, and $\mathrm{ch}=$ check star.

Adelman et al. (2001) used consequences 1 and 2. 20 Eri (Adelman 2000) and 108 Aqr (Adelman 1999) are fast rotating stars which apparently act in a similar manner. The behavior of CU Vir (Pyper et al. 1998) may include a precession-like component (Stepien 1998). Consequence 3 remains to be observed.

MW Vul, which is a sharp-lined rather than a rapidly rotating star, should have a near spherical photosphere. Thus consequences of type 1 will be minimal. This is the most likely explanation of what is observed is the result of the local energy distribution changes substantially over the photosphere. Shore \& Adelman (1974) anticipated precession to be seen in broadlined $\mathrm{mCP}$ stars unless there was a close binary companion to produce a forced body precession. The orbit of the detected companion needs to be measured and a careful search for a closer companion needs to be made. It would also be useful to obtain additional measurements of its magnetic field to see if it is variable with the photometric period as anticipated.

\section{3. $H R 9017$}

HR 9017 (HD 223 358, V650 Cas, HIP 117430) is a visual binary (Hartkopf et al. 2000). Winzer (1974) found it was a photometric variable with an amplitude of $0.02 \mathrm{mag}$ in $U$ based on 15 sets of $U B V$ observations. His period was 0.9135 days. Later Burke \& Barr (1981) obtained an additional 19 sets of $U B V$ values. They found a period of 1.092 days and an amplitude of $0.03 \mathrm{mag}$ in $U$. The two periods are nearly inverses of one another.

During the 2002-03 and 2003-04 observing seasons 53 sets of $u v b y$ observations were made (see Table 4). A period of 0.92738 days was derived using the Scargle periodogram. It corresponded to the frequency with greatest signal-to-noise ratio. The inverse of the adopted frequency is not quite as strong and does not result in as nice light curves especially for $u$. It is not possible to use the two sets of data to derive a better period as the two sets of values are separated by a long period of time. Hipparcos photometry (ESA 1997) shows a range of 0.03 mag which does not necessary indicate variability and Celestia 2000 (ESA 1998) notes that its variability is spurious due to duplicity.

Figure 3 shows the $u v b y$ photometry plotted as a function of the derived ephermis
HJD (light maximum) $=2452453.7021( \pm 0.0015)+$ $0.92738( \pm 0.0015) E$.

The observed amplitudes are $0.025 \mathrm{mag}$ in $u$ and around 0.01 mag for $b, v$, and $y$. The light curves are best described as in phase. For $u$ the maximum and the minimum appear to be symmetric.

\section{The HgMn Star $46 \rho$ Aqr}

For the $\mathrm{HgMn}$ stars, which are non-magnetic CP stars, a question of particular importance is whether there exist any nonintrinsically constant class members, which are not located in the variability strip. On the basis of their Hipparcos photometry Adelman (1998) noted that several HgMn stars that might be possible variables including $46 \rho$ Aqr (HR 8512, HD 211838 , HIP 110273).

Aikman (1976) found $46 \rho$ Aqr had a variable radial velocity. Stickland \& Weatherby (1983) obtained an orbital solution with an inhomogenous set of archival data with a period of 220.41 days. Celestia 2000 (ESA 1998) indicates that binarity was found by the Hipparcos satellite. Hipparcos photometry (ESA 1997) shows for 54 measurements a mean magnitude of 5.3365 mag with a standard error of $0.0015 \mathrm{mag}$ and a range of $0.02 \mathrm{mag}$.

$46 \rho$ Aqr was observed nine times during the 2002-03 observing season in Spring 2003 and 35 times during the 2003-04 observing season in Fall 2003. The mean and standard deviation of the mean for the $u, v, b$, and $y$ values both for the variable-comparison and for the check-comparison stars are given in Table 5 with the photometric values in Table 6 . The ch-c values are offset especially for $b$ and $y$ and superimposed on this are larger mean differences of the $\mathrm{v}$-c values are seen for $v, b$, and $y$. As the behavior of the v-c values is not seen in other $\mathrm{HgMn}$ stars, it suggests that this is due to the secondary star being variable. The offset of the ch-c values suggests that the comparison or the check star is slowly variable. As this is a smaller effect than that seen for $\mathrm{v}$-c for most colors, the latter is probably real and needs further confirmation even though it is probably not related to the primary HgMn star. The Hipparcos photometry of the comparison and check stars (ESA 1998) indicates that both are equally stable. But as $\sigma$ Aqr is a hot Am star, it is expected to be constant. Thus $51 \mathrm{Aqr}$ is more likely to be variable. But as one of these stars is $3^{\circ}$ North and the other $3^{\circ}$ South of the variable, a slight change in the extinction 


\section{HR 9017}
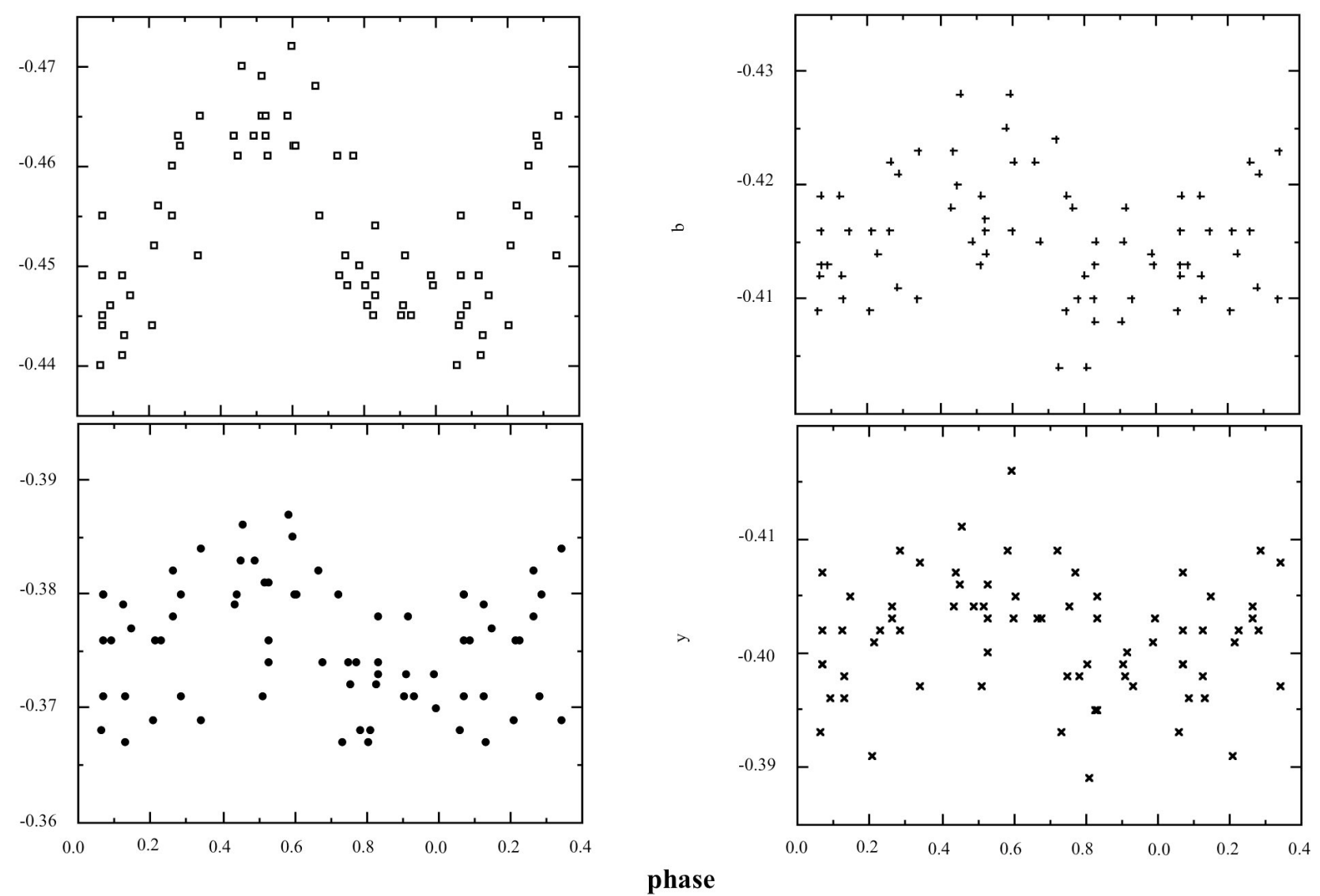

Fig. 3. uvby photometry of the mCP star HR 9017 as a function of the ephemeris $\operatorname{HJD}($ light maximum) $=2452453.7021+0.92738 E$.

(the data were reduced with mean extinction coefficients) could also play a role in the ch-c changes, but the offset in $u$ indicates that other factor are in play.

\section{Final comments}

The study of $\rho 46$ Aqr is the last of the HgMn stars which can be observed with the FCAPT that Adelman (1998) noted might be variable in Hipparcos photometry. As none of those which are single was variable, it appears that the possible variability seen was due to the companion.

The changes in the light curves of MW Vul indicate that further observations of this star are needed. To find the period of the secondary variability will require complete light curves taken every year or two. Comparison of the various yearly light curves suggest it takes two years or more to see changes. The magnetic field value of Babcock needs to be verified. Further if MW Vul is truly precessing, its apparent rotational velocity should change during the precessional period. It requires high resolution spectra to track this effect. As this star was not expected to show the variability characteristic of a precessing star, this raises theoretical issues as well as indicates that other stars with similar behavior may be found.

Acknowledgements. This work was supported in part by NSF grant AST-0071260. We appreciate the continuing efforts of Louis J. Boyd,
Robert J. Dukes, Jr., and George P. McCook to keep the FCAPT operating properly. This research has used the SIMBAD database, operated at the CDS, Strasbourg, France.

\section{References}

Adelman, S. J. 1975, ApJ, 195, 397

Adelman, S. J. 1982, A\&AS, 49, 668

Adelman, S. J. 1997, A\&AS, 125, 65

Adelman, S. J. 1998, A\&AS, 132, 93

Adelman, S. J. 1999, Baltic Astron., 8, 369

Adelman, S. J. 2000, A\&AS, 146, 13

Adelman, S. J. 2002, Baltic Astron., 11, 475

Adelman, S. J., \& Knox, J. R., Jr. 1994, A\&AS, 103, 1

Adelman, S. J., Malanushenko, V., Ryabchikova, T. A., \& Savanov, I. 2001, A\&A, 375, 982

Adelman, S. J., \& Pyper, D. M. 1993, A\&AS, 101, 393

Aikman, G. C. L. 1976, Publ. Dom. Astrophys. Obs., 14, 379

Babcock, H. W. 1958, ApJS, 3, 141

Bartolini, C., Forschini, R., \& Piccioni, A. 1974, Inf. Bull Var. Stars, 948

Burke, E. W., Jr., \& Barr, T. H. 1981, PASP, 93, 344

ESA 1997, The Hipparcos and Tycho Catalogs, SP-1200

ESA 1998, The Hipparcos and Tycho Catalogs, Celestia 2000, SP-1220

Hartkopf, W. I., Mason, B. D., \& Worley, C. E. 2000, Fifth Catalog of Orbits of Visual Binary Stars (Washington, DC: US Naval Observatory) 
Hoffleit, D. 1982, The Bright Star Catalogue, 4th edition (New Haven, CT: Yale University Observatory)

Hoffleit, D., Saladyga, M., \& Wlasuk, P. 1983, A Supplement to the Bright Star Catalogue (New Haven, CT: Yale University Observatory)

Horne, J. H., \& Baliunas, S. L. 1986, ApJ, 302, 757

Lopez-Garcia, Z., \& Adelman, S. J. 1994, A\&AS, 107, 353

Michaud, G., \& Proffitt, C. R. 1993, in Peculiar Versus Normal Phenomena in A-Type and Related Stars, ed. M. M. Dworetsky, F. Castelli, \& R. Faraggiana (San Francisco: ASP), ASP Conf. Proc., 44,439

Musielok, B., Lange, D., Schöneich, W., et al. 1980, Astron. Nach, 301,71
Pyper, D. M., Ryabchikova, T., Malanushenkov, V., et al. 1998, A\&A, 339,822

Preston, G. W. 1971, ApJ, 164, 309

Ryabchikova, T. A., Davidova, E. S., \& Adelman, S. J. 1990, PASP, 102,581

Scargle, J. D. 1982, ApJ, 263, 835

Shore, S. N., \& Adelman, S. J. 1974, ApJ, 191, 165

Stepien, K. 1998, A\&A, 337, 754

Stickland, D. J., \& Watherby, J. 1983, A\&AS, 57, 55

Winzer, J. E. 1974, Ph.D. Thesis, University of Toronto

Wolff, S. C., \& Preston, G. W. 1978, ApJS, 37, 371 